\title{
Nucleation modeling of the Antarctic stratospheric CN layer and derivation of sulfuric acid profiles
}

\author{
Steffen Münch ${ }^{1, \mathrm{a}}$ and Joachim Curtius ${ }^{1}$ \\ ${ }^{1}$ Institute for Atmospheric and Environmental Sciences, Goethe University Frankfurt am Main, \\ 60323 Frankfurt am Main, Germany \\ ${ }^{a}$ now at: Institute for Atmospheric and Climate Science, ETH Zurich, 8092 Zurich, Switzerland
}

Correspondence to: Steffen Münch (steffen.muench@env.ethz.ch)

Received: 5 July 2016 - Discussion started: 7 July 2016

Revised: 2 May 2017 - Accepted: 4 May 2017 - Published: 22 June 2017

\begin{abstract}
Recent analysis of long-term balloon-borne measurements of Antarctic stratospheric condensation nuclei $(\mathrm{CN})$ between July and October showed the formation of a volatile $\mathrm{CN}$ layer at $21-27 \mathrm{~km}$ altitude in a background of existing particles. We use the nucleation model SAWNUC to simulate these $\mathrm{CN}$ in subsiding air parcels and study their nucleation and coagulation characteristics. Our simulations confirm recent analysis that the development of the $\mathrm{CN}$ layer can be explained with neutral sulfuric acid-water nucleation and we show that outside the $\mathrm{CN}$ layer the measured $\mathrm{CN}$ concentrations are well reproduced just considering coagulation and the subsidence of the air parcels. While ion-induced nucleation is expected as the dominating formation process at higher temperatures, it does not play a significant role during the $\mathrm{CN}$ layer formation as the charged clusters recombine too fast. Further, we derive sulfuric acid concentrations for the $\mathrm{CN}$ layer formation. Our concentrations are about 1 order of magnitude higher than previously presented concentrations as our simulations consider that nucleated clusters have to grow to $\mathrm{CN}$ size and can coagulate with preexisting particles. Finally, we calculate threshold sulfuric acid profiles that show which concentration of sulfuric acid is necessary for nucleation and growth to observable size. These threshold profiles should represent upper limits of the actual sulfuric acid outside the $\mathrm{CN}$ layer. According to our profiles, sulfuric acid concentrations seem to be below midlatitude average during Antarctic winter but above midlatitude average for the $\mathrm{CN}$ layer formation.
\end{abstract}

\section{Introduction}

Atmospheric aerosol particles are of interest due to their various influences on radiation, clouds, chemistry, and air quality. Condensation nuclei counters measure the number concentration of aerosol particles by growing them to optically detectable sizes by condensation (e.g., McMurry, 2000). Therefore, condensation nuclei $(\mathrm{CN})$ are defined as all aerosol particles that are large enough to be measured by a $\mathrm{CN}$ counter operating at a given supersaturation, which typically can measure particles with diameters larger than $\sim 10 \mathrm{~nm}$. New particle formation to supply the particles for the stratosphere mostly occurs in the tropical tropopause layer (Brock et al., 1995; Thomason and Peter, 2006; Weigel et al., 2011). The particles are then distributed in the lower stratosphere and constitute the stratospheric aerosol layer (Junge et al., 1961). The particles are sulfuric acid-water droplets (Arnold et al., 1998; Deshler, 2008; Junge et al., 1961), and if they are completely volatile, they are assumed to be formed by ion-induced or neutral homogeneous nucleation of sulfuric acid and water (binary nucleation). Binary nucleation occurs at low temperatures and high sulfuric acid concentrations. In the stratosphere the lowest temperatures are found in the polar vortex of the winter hemisphere. In the Antarctic polar vortex a background $\mathrm{CN}$ concentration of $\sim 10 \mathrm{~cm}^{-3}$ is found (Campbell and Deshler, 2014). Volatility measurements indicate that more than half of them have a nonvolatile core which could be meteoric material (Campbell and Deshler, 2014; Curtius et al., 2005). Sulfuric acid is expected to condense on these CN (Borrmann et al., 2010; Murphy et al., 1998, 2014) and the formation rate of gaseous 
sulfuric acid should be very small during polar night. Therefore, nucleation is not expected to occur in the polar vortex.

Contrary to this expectation, Rosen and Hofmann (1983) first observed an increase in volatile $\mathrm{CN}$ at $25-30 \mathrm{~km}$ altitude during winter at Laramie, Wyoming $\left(41^{\circ} \mathrm{N}\right)$. They assumed the $\mathrm{CN}$ to be freshly nucleated sulfuric acid-water particles with the polar stratosphere as the source region. Above McMurdo Station, Antarctica $\left(78^{\circ} \mathrm{S}\right)$, Hofmann and Rosen (1985) also observed an increased CN concentration between 20 and $25 \mathrm{~km}$ after sunrise (CN layer). To check if the occurrence of this $\mathrm{CN}$ layer was an annual polar phenomenon, further measurements were performed that also observed the formation of a CN layer after sunrise (e.g., Hofmann, 1990, at Kiruna, Sweden $\left.\left(68^{\circ} \mathrm{N}\right)\right)$. Therefore, sulfuric acid production by sunlight after the end of the polar night was suggested as the nucleation source.

Based on these observations, modeling studies began to investigate the formation of the CN layer. Hamill et al. (1990) calculated nucleation rates indicating that binary nucleation could occur in the polar winter stratosphere if sulfuric acid concentrations were high enough. Zhao et al. (1995) developed a one-dimensional (altitude) aerosol model that showed that the transformation of carbonyl sulfide (OCS) to $\mathrm{SO}_{2}$ and further oxidation of $\mathrm{SO}_{2}$ to sulfuric acid are too slow to reproduce the observed $\mathrm{CN}$ increase. They could only reproduce the formation of the $\mathrm{CN}$ layer when they added downward transport of $\mathrm{SO}_{2}$ from the mesosphere inside the polar vortex. Mills et al. $(1999,2005)$ presented modeling with a two-dimensional (altitude and latitude) aerosol model that was able to reproduce the formation of the $\mathrm{CN}$ layer when including production of mesospheric $\mathrm{SO}_{2}$ by photolysis of sulfuric acid and $\mathrm{SO}_{3}$ (see also Vaida et al., 2003).

In summary, and contrary to the initial expectation, nucleation seems to occur in the polar stratosphere. During polar winter, more $\mathrm{SO}_{2}$ is transported downward inside the polar vortex without being oxidized by photochemical reactions. After sunrise, this $\mathrm{SO}_{2}$ is oxidized to sulfuric acid, which initiates nucleation and forms the volatile $\mathrm{CN}$ layer despite the presence of nonvolatile background $\mathrm{CN}$.

More recently, Campbell and Deshler (2014) presented a long-term record of stratospheric balloon-borne $\mathrm{CN}$ measurements that were performed 2-3 times a year during winter from 1986 until 2010 above McMurdo Station, Antarctica $\left(78^{\circ} \mathrm{S}\right)$. They present monthly averaged $\mathrm{CN}$ concentration and temperature profiles which capture the unperturbed $\mathrm{CN}$, with concentrations around $10-20 \mathrm{~cm}^{-3}$ in June/July as well as the development of the $\mathrm{CN}$ layer at $21-27 \mathrm{~km}$, with concentrations increasing to $100 \mathrm{~cm}^{-3}$ from August until October during sunrise and warming. Campbell and Deshler (2014) also presented volatility measurements of the $\mathrm{CN}$ showing that in general more than half of the $\mathrm{CN}$ have a nonvolatile core except in the $\mathrm{CN}$ layers where they observe significant and rapid formation of new particles that are completely volatile. Additionally, Campbell et al. (2014) used a three-dimensional chemistry climate model (English et al.,
2011; Hurrell et al., 2013) to reveal the global extent of the CN layer.

Campbell and Deshler (2014) describe a method where they derive an Antarctic sulfuric acid profile from the measured $\mathrm{CN}$ by inverting the neutral binary nucleation equation. They used the difference between the $\mathrm{CN}$ before sunrise and two weeks after sunrise averaged over all years to derive a nucleation rate for all altitudes from which they derived the corresponding sulfuric acid. This profile is useful, for example, for evaluating global models (Campbell et al., 2014) as no Antarctic sulfuric acid measurements exist. However, Campbell and Deshler (2014) and Campbell et al. (2014) also note that their derived profile might be an underestimation as their method does not consider the particles smaller than their experimental $\mathrm{CN}$ detection threshold particle size, losses to preexisting particles, and ion-induced nucleation.

We find this approach of deriving a sulfuric acid profile from the measured $\mathrm{CN}$ intriguing. Here we use the nucleation model SAWNUC that simulates small particles, ion-induced nucleation, coagulation, and losses to preexisting particles. We model the Antarctic CN layer based on the observations of Campbell and Deshler (2014) and derive Antarctic stratospheric sulfuric acid profiles.

\section{Methods}

\subsection{The SAWNUC model}

The SAWNUC (Sulfuric Acid Water NUCleation; Lovejoy et al., 2004) model simulates binary sulfuric acid water neutral and ion-induced nucleation. SAWNUC uses thermodynamic stabilities that are based on experimental values and quantum chemical calculations (Lovejoy and Curtius, 2001; Froyd and Lovejoy, 2003a, b; Hanson and Lovejoy, 2006), and it explicitly simulates step-by-step addition of sulfuric acid molecules in linear size bins for cluster sizes below $2 \mathrm{~nm}$. Above $2 \mathrm{~nm}$ particle concentrations are collected in geometric size bins. Here we simulate 30 geometric size bins with a scale factor of 1.7 , ranging up to about $400 \mathrm{~nm}$ for neutral and negatively charged clusters. For each size bin, SAWNUC can simulate condensation and evaporation of sulfuric acid, coagulation with neutral clusters, recombination of negative clusters with positive ions, and losses to preexisting particles. SAWNUC has been previously described and used (among others) by Lovejoy et al. (2004), Ehrhart and Curtius (2013), Kürten et al. (2015), Ehrhart et al. (2016), and its parameterized version, PARNUC (Kazil and Lovejoy, 2007), is used in Kirkby et al. (2011).

For this study, we extended the SAWNUC model. As in Kürten et al. (2015), coagulation rates between neutral clusters are now calculated including van der Waals forces according to Chan and Mozurkewich (2001). We redesigned the model code to allow for changes in ambient conditions during a simulation and added the ability to perform mul- 

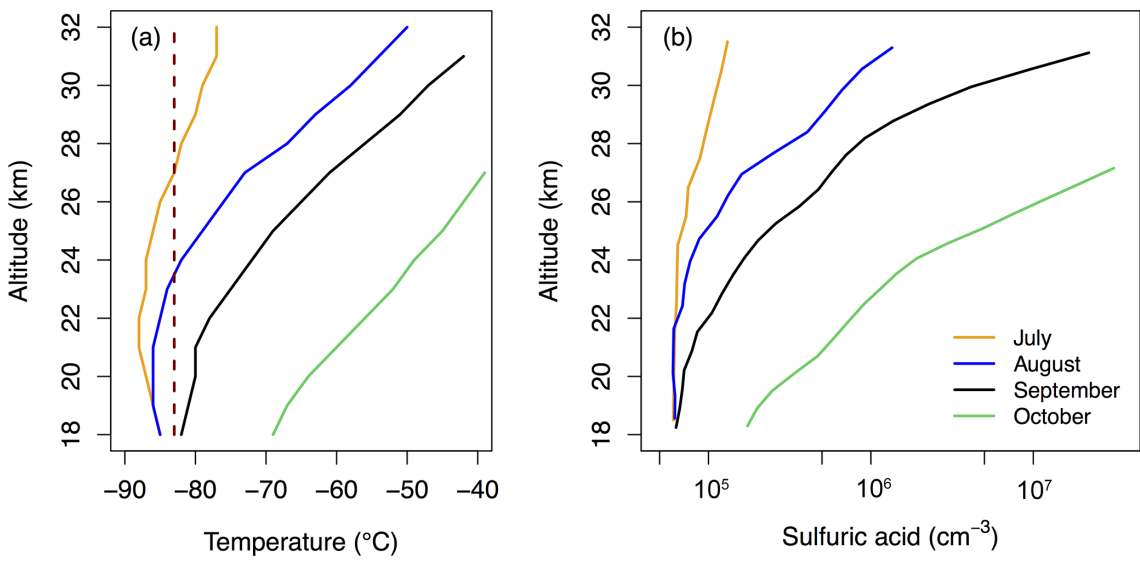

Figure 1. Temperatures (a) during Antarctic winter above McMurdo Station, Antarctica $\left(78^{\circ} \mathrm{S}\right)$, as presented in Campbell and Deshler (2014). The dashed line shows the lower temperature limit for which the SAWNUC model is valid and at which lower temperatures were kept fixed. In (b), corresponding sulfuric acid profiles are shown that lead to a $10 \% \mathrm{CN}$ increase by nucleation and growth to observable size during 1 month. For these nucleation threshold profiles, we assume a monodisperse $\mathrm{CN}$ background of $10 \mathrm{~cm}^{-3}$ with $100 \mathrm{~nm}$ diameter at all altitudes $(18-32 \mathrm{~km})$ for every month (July-October).

tiple simulations within one program run. We do not use SAWNUC's procedure to represent losses to preexisting particles by a single surface area loss term, but instead we now fully simulate preexisting particles as initial particle concentrations. For this study, the basic processes simulated by SAWNUC are condensation and evaporation of sulfuric acid and coagulation for every size bin. Condensation and evaporation of sulfuric acid are the dominating processes for the formation of new particles, while coagulation and condensation of sulfuric acid, if present, determine growth and number reduction of existing particles.

\subsection{Ambient parameters}

To perform a regular SAWNUC simulation for a given region of the Antarctic stratosphere, the temperature, pressure, ion pair production rate, relative humidity, and sulfuric acid concentration are required. Particle concentrations and sizes are the model output at every time step. When inverting SAWNUC, the particle concentrations are required to derive the sulfuric acid concentrations.

Temperatures above Antarctica are taken from Campbell and Deshler (2014), and those temperatures that are below $190 \mathrm{~K}$ (maximum $5 \mathrm{~K}$ below), which is SAWNUC's lower limit of the temperature range, are fixed at $190 \mathrm{~K}$. This introduces some uncertainty which is estimated in our sensitivity test of a $5 \mathrm{~K}$ temperature increase (Sect. 3.3). Altitudes are converted to pressures according to the global modeling of Campbell et al. (2014). The ionization rate of the Antarctic stratosphere in August-September 2010 was $3 \times 10^{5}$ ion pairs per gram of air and second (Ilya Usoskin, personal communication, 2013 according to Usoskin et al., 2011), which converts to, for example, $\sim 10$ ion pairs $\mathrm{cm}^{-3} \mathrm{~s}^{-1}$ at $200 \mathrm{~K}$ and $20 \mathrm{hPa}$. The water vapor profile for July increases linearly from 3.0 to $6.0 \mathrm{ppm}$ between 18 and $25 \mathrm{~km}$, while remaining at a constant value of $6.0 \mathrm{ppm}$ up to $32 \mathrm{~km}$ based on the Microwave Limb Sounder (Waters et al., 2006) and hygrometer (Vömel et al., 1995) measurements in Fig. 7a in Campbell and Deshler (2014). The water vapor mixing ratio is kept constant during the subsidence of the simulated air parcels (see below).

CN concentrations are taken from Campbell and Deshler (2014). The measured $\mathrm{CN}$ are then compared with the simulated $\mathrm{CN}$ by summing over all simulated particles with diameters above $20 \mathrm{~nm}$, as Campbell and Deshler (2014) reported a detection limit of their $\mathrm{CN}$ counters of $6-20 \mathrm{~nm}$ diameter. As we do not know the exact size of the measured $\mathrm{CN}$, we assume the initial preexisting $\mathrm{CN}$ to have a diameter of $100 \mathrm{~nm}$ (see below), but we also perform sensitivity studies assuming different sizes in Sect. 3.3. We simulate them as pure sulfuric acid-water particles but as temperatures are too low for significant evaporation, they could also include a nonvolatile core.

\section{Results}

\subsection{CN simulations and sulfuric acid profiles}

We start our simulations with a simplified reference case where we assume for all altitudes $(18-32 \mathrm{~km})$ and for every month (July-October) a constant monodisperse background $\mathrm{CN}$ concentration of $10 \mathrm{~cm}^{-3}$ with a diameter of $100 \mathrm{~nm}$. For this reference case, we do not simulate the region above $31 \mathrm{~km}$ in September and above $27 \mathrm{~km}$ in October, as high temperatures lead to evaporation of the initial background $\mathrm{CN}$ and complicate the interpretation. For all other altitudes and months, we simulate 1 month with constant ambient conditions chosen according to Sect. 2.2. We use the tempera- 

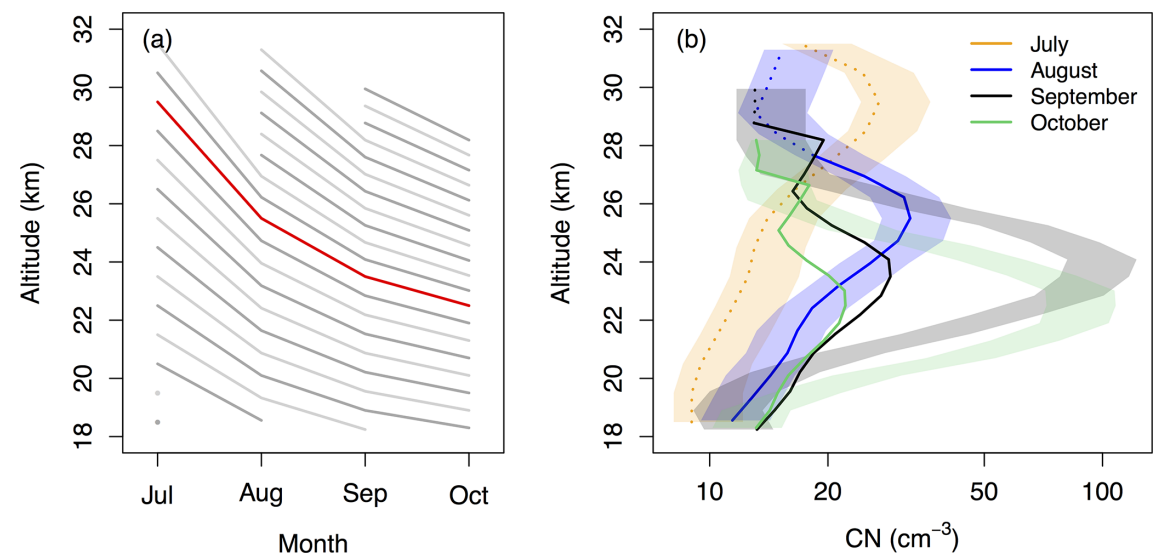

Figure 2. Air parcel subsidence trajectories (a) and simulated CN (monthly mean) without gaseous sulfuric acid being present (b). The uncertainty ranges of the measured CN presented in Campbell and Deshler (2014) are shown as shaded areas in (b) for comparison. The trajectories of the simulated air parcels were placed around the subsidence of the measured $\mathrm{CN}$ maximum (red). In the simulation, the ambient conditions are kept constant during each month. For the first month of each trajectory, the $\mathrm{CN}$ concentrations (dotted) are chosen based on Campbell and Deshler (2014). In the following months, the simulated CN concentrations (solid) are the result of only coagulation and air volume compression, as there is no gaseous sulfuric acid present.
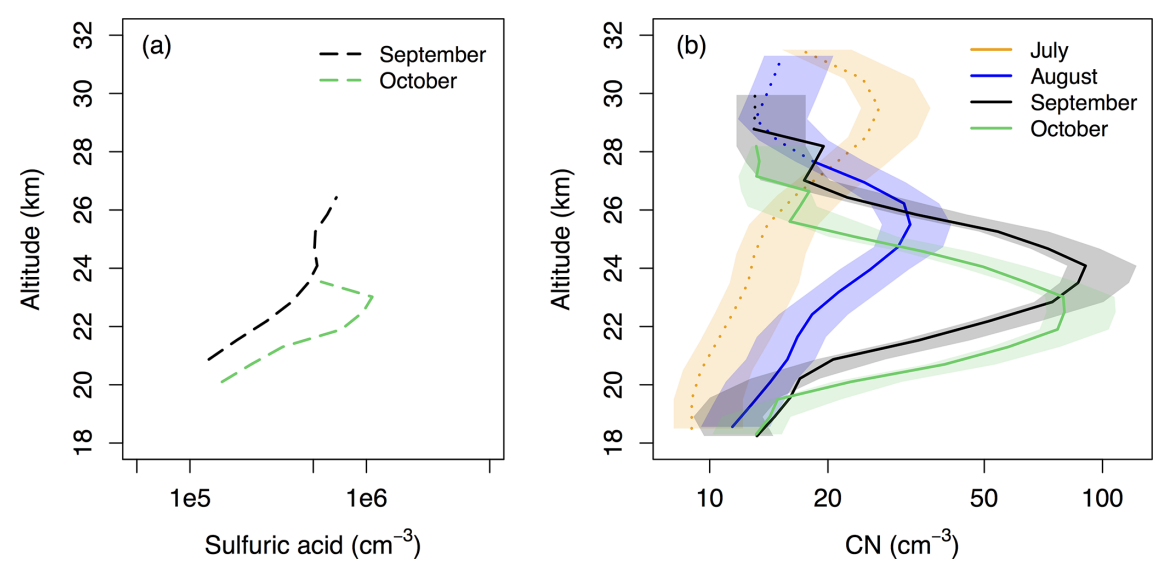

Figure 3. CN layer gaseous sulfuric acid profiles (a) and the simulated $\mathrm{CN}$ using these profiles (b). We derive the sulfuric acid if the simulated $\mathrm{CN}$ concentrations in Fig. $2 \mathrm{~b}$ are too low without gaseous sulfuric acid being present and therefore nucleation and condensational growth are needed to simulate the $\mathrm{CN}$ layer. As in Fig. 2b, the dotted lines are the initial $\mathrm{CN}$ based on observations and the solid lines are the simulated CN. The uncertainty ranges of the measured CN from Campbell and Deshler (2014) are shown as shaded areas for comparison.

tures reported by Campbell and Deshler (2014) which are reproduced in Fig. 1a. We set the background concentration of $10 \mathrm{CN} \mathrm{cm}^{-3}$ as initial particle concentrations at the beginning of the month and simulate the month without gaseous sulfuric acid being present. The $\mathrm{CN}$ concentration then reduces somewhat over time as the particles coagulate. Then, we simulate the month again and derive the gaseous sulfuric acid concentration that leads to a $10 \%$ higher monthly mean $\mathrm{CN}$ concentration. This we term the nucleation threshold profile for sulfuric acid as it defines the minimum gaseous sulfuric acid that leads to nucleation and growth to observable $\mathrm{CN}$ size of about 1 additional $\mathrm{CN}$ per $\mathrm{cm}^{3}$ and month in a background of 10 preexisting $\mathrm{CN} \mathrm{cm}^{-3}$. Note that even though the idealized assumption of zero gaseous sulfuric acid is unphysical, it helps us to fully isolate and understand the processes that occur. All results of this study hardly change if we assume a minimum sulfuric acid concentration of $10^{4} \mathrm{~cm}^{-3}$ instead of zero sulfuric acid. Although the $10^{4} \mathrm{~cm}^{-3}$ sulfuric acid would be more realistic, we preferred the idealized choice of zero sulfuric acid to be able to completely decouple the processes.

The nucleation threshold sulfuric acid profiles are shown in Fig. 1b. Their shapes are similar to the temperature profiles because temperature, sulfuric acid, and losses to preexisting particles mainly determine the nucleation rate. As the preexisting particle concentrations are the same and we target almost the same nucleation rate everywhere, the temperature determines the derived sulfuric acid concentration, and the 


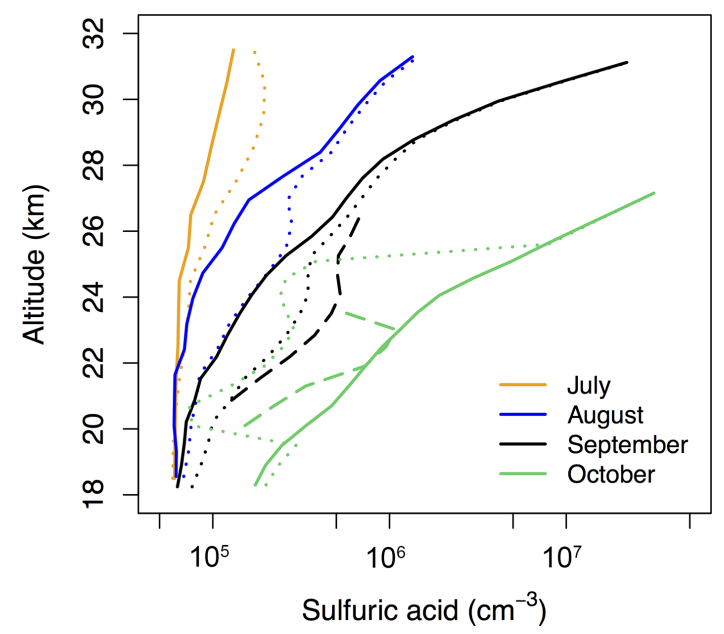

Figure 4. Combination of the nucleation threshold sulfuric acid profiles from Fig. $1 \mathrm{~b}$ (solid) and the $\mathrm{CN}$ layer sulfuric acid profiles from Fig. 3a (dashed). Additionally, we show sulfuric acid profiles that cause a $\mathrm{CN}$ increase in our $\mathrm{CN}$ simulation of Fig. 3b (dotted) which should represent upper limits of the Antarctic winter stratospheric sulfuric acid outside the $\mathrm{CN}$ layer.

nucleation threshold profiles consequently increase with increasing temperature. However, the water vapor concentration also has a small influence on the derived profiles as can be seen, for example, in July below $27 \mathrm{~km}$, where the temperature is fixed to $190 \mathrm{~K}$ but the derived profile still varies slightly.

We continue by studying how the measured $\mathrm{CN}$ of Campbell and Deshler (2014) coagulate outside the $\mathrm{CN}$ layer. Therefore, we drop the assumption of $10 \mathrm{CN} \mathrm{cm}^{-3}$ from the reference case and simulate the $\mathrm{CN}$ inside air parcels that subside in the polar vortex. The monthly average $\mathrm{CN}$ profiles presented in Fig. 1c in Campbell and Deshler (2014) are our basis for the air parcel subsidence trajectories. Campbell and Deshler (2014) suggested air subsidence inside the polar vortex as the explanation for the subsidence of the $\mathrm{CN}$ maximum from July to October. We follow their suggestion and assume that the $\mathrm{CN}$ maximum of each month resides in a single subsiding air parcel, and place the other air parcel trajectories around this $\mathrm{CN}$ maximum trajectory (Fig. 2a; description in Sect. S2 in the Supplement). Note that the CN maximum subsidence trajectory is an average over 24 years of measurements and therefore our air parcel trajectories represent idealized descents. The pathways outside the $\mathrm{CN}$ layer are just rough estimates. However, our trajectories seem reasonable as Hardiman et al. (2013) find residual vertical velocities of about $0.6 \mathrm{~mm} \mathrm{~s}^{-1}\left(\approx 1.5 \mathrm{~km}\right.$ per month) at $80^{\circ} \mathrm{S}$ and $70 \mathrm{hPa}$ that increase with height in their analysis of the Brewer-Dobson circulation in CMIP5 simulations. For an air parcel simulation, we set the ambient conditions at the beginning of each month according to Sect. 2.2 and keep them constant for the whole month. We simulate and compare the values on a monthly basis as the measured input and target values (temperature and $\mathrm{CN}$ ) are monthly averages as well. For the first month of each parcel simulation, we use the measurements of Campbell and Deshler (2014) to determine the initial $\mathrm{CN}$ concentration and subsequently simulate this month. As we still assume no gaseous sulfuric acid being present, coagulation is the only process taking place which reduces the $\mathrm{CN}$ by some amount. We choose the initial $\mathrm{CN}$ concentration so that the mean $\mathrm{CN}$ concentration in the first month matches the measurements. After the first month, we let the model run free, still assuming zero gaseous sulfuric acid. Therefore, the only two effects on $\mathrm{CN}$ concentration are a decrease due to coagulation and an increase when the ambient conditions change between the months. The latter results from an air volume expansion by temperature increase and an air volume compression by pressure increase which is mostly dominated by the pressure effect and consequently results in a net $\mathrm{CN}$ concentration increase.

Figure $2 b$ shows the simulated $\mathrm{CN}$ without sulfuric acid being present and therefore no nucleation. The uncertainty ranges of the measured $\mathrm{CN}$ from Campbell and Deshler (2014) are shown for comparison $(-10$ to $+35 \%)$. As the first value of every simulated air parcel is chosen based on the observations, the $\mathrm{CN}$ in July and at the top in August and September are identical with the measurements (dotted). In the following months, the $\mathrm{CN}$ result from our simulation (solid). In August, subsidence and air compression of the July $\mathrm{CN}$ dominate over coagulation throughout the simulated profile and lead to higher $\mathrm{CN}$ concentrations. Thereby, the measured $\mathrm{CN}$ can be fully reproduced within the uncertainty range without nucleation of new particles being necessary. In September and October, the modeled CN concentrations at $20-27 \mathrm{~km}$ are too small under a zero gaseous sulfuric acid condition, and the $\mathrm{CN}$ layer is not simulated.

In September, above the CN layer at $\sim 28 \mathrm{~km}$, too many $\mathrm{CN}$ are simulated even without any gaseous sulfuric acid being present (Fig. 2b). This is the result of an air volume compression in the subsiding air parcels from $31 \mathrm{~km}$ in August to $28 \mathrm{~km}$ in September which increases the $\mathrm{CN}$ concentration by $\sim 60 \%$. Here, coagulation is not efficient enough to reduce the monthly mean $\mathrm{CN}$ to the observed value. The high October $\mathrm{CN}$ at $\sim 26.5 \mathrm{~km}$ are then a result of the high September values. If the August $\mathrm{CN}$ concentration at $\sim 31 \mathrm{~km}$ is reduced by about one-third, the simulated CN in September and October would be within the measurement range.

It is important to understand how much sulfuric acid is necessary to form the $\mathrm{CN}$ layer, and thus reproduce the observations. Using the same method as before, we now derive the amount of sulfuric acid needed to match the simulated and measured $\mathrm{CN}$. This sulfuric acid causes nucleation of new particles, growth of these new particles to $\mathrm{CN}$ size, and growth of existing $\mathrm{CN}$.

Figure 3a shows the sulfuric acid profiles that are necessary to form the $\mathrm{CN}$ layer and reproduce the observations (termed "CN layer profiles"). Sulfuric acid is needed 
in September at $21-26 \mathrm{~km}$ to form the CN layer and in October at $20-24 \mathrm{~km}$ to prevent the $\mathrm{CN}$ layer from decreasing too fast by coagulation. Figure $3 \mathrm{~b}$ shows the simulated $\mathrm{CN}$ using the derived sulfuric acid profiles, and as expected the measured $\mathrm{CN}$ profiles are reproduced for all months at almost all altitudes.

For a complete interpretation of the processes in the $\mathrm{CN}$ layer we combine our nucleation threshold profiles and $\mathrm{CN}$ layer profiles in Fig. 4 (solid and dashed). Additionally, we derive the sulfuric acid concentrations that lead to a $\mathrm{CN}$ increase in our simulation of the observed CN (Fig. 3b) and include them in Fig. 4 (dotted). We use the same method as for our nucleation threshold profiles (deriving the amount of sulfuric acid that leads to a $10 \% \mathrm{CN}$ increase), but now with the simulated $\mathrm{CN}$ as background. Note that outside of the $\mathrm{CN}$ layer, these profiles represent only upper limits for the gaseous sulfuric acid in the atmosphere as neither the observations nor our simulations indicate nucleation in these areas.

In July, August, and September, the upper limit profiles (dotted) show the sulfuric acid that is necessary for nucleation and growth to $\mathrm{CN}$ size and leads to additional $10 \% \mathrm{CN}$ within 1 month. The concentrations are higher than our nucleation threshold profiles (solid) because we have a higher concentration of preexisting $\mathrm{CN}$ compared to the $10 \mathrm{~cm}^{-3}$ in the reference case. Therefore, more small clusters are lost by coagulation with large $\mathrm{CN}$. Here nucleation is in competition with losses to preexisting particles (Ehrhart and Curtius, 2013). In September, the sulfuric acid profile that forms the $\mathrm{CN}$ layer (dashed) has higher concentrations than both the nucleation profile (solid) and the upper limit profile (dotted). This means that new particles are nucleating and that more than the $10 \%$ additional $\mathrm{CN}$ of the upper limit case have to form. However, as the nucleation rate is very sensitive to changes in sulfuric acid, the derived $\mathrm{CN}$ layer sulfuric acid concentrations are not much higher than the upper limit concentrations.

In October in the area of the $\mathrm{CN}$ layer, however, the upper limit profile (dotted) and the $\mathrm{CN}$ layer formation profile (dashed) are both lower than the nucleation profile (solid), showing that no new particles have to nucleate. Instead, small particles that still exist from the nucleation event in September can grow above the $\mathrm{CN}$ counting threshold, which requires less sulfuric acid than nucleation and growth of new CN. Therefore, the history of the nucleation event in September allows for a $\mathrm{CN}$ increase without new particle formation in October.

In the following sensitivity studies we show and discuss only the nucleation threshold and the $\mathrm{CN}$ layer profiles to avoid overloaded figures, but the conclusions for the upper limit profiles are analogous to the other profiles.

\subsection{Ion-induced nucleation}

Figure 5 shows the impacts of ion-induced nucleation on the derived sulfuric acid profiles by removing all ions from the

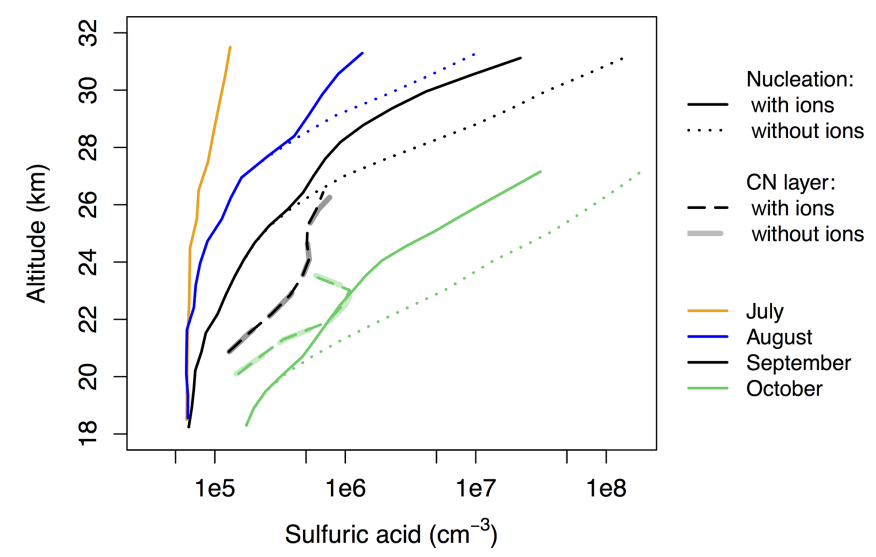

Figure 5. Comparison of the nucleation threshold sulfuric acid profiles derived including ion-induced nucleation (solid lines) and without simulating ions (dotted lines). At low sulfuric acid concentrations the derived profiles do not change. The $\mathrm{CN}$ layer profiles also hardly change (thick dashed lines; grey and light green are without ions and black and green are with ions, but they are almost identical).

simulations and then comparing the derived profiles to those that included ions. In areas with low sulfuric acid concentrations, removing the ions has nearly no effect on the derived profiles; however, in areas with higher sulfuric acid concentrations the derived profiles increase by almost an order of magnitude. At low sulfuric acid concentrations, the small clusters are not growing fast enough by condensation. Negatively charged clusters recombine too early with positively charged ions and therefore are too small to overcome the nucleation barrier of neutral nucleation. At higher sulfuric acid concentrations, ion-induced nucleation occurs as expected. The charged clusters grow larger than the critical size before they recombine and increase the nucleation rate. Thus to create the same number of $\mathrm{CN}$ without ions, more sulfuric acid is required than if ions are present.

For the nucleation threshold profiles with $10 \mathrm{~cm}^{-3}$ background $\mathrm{CN}$, ion-induced nucleation starts to occur at sulfuric acid concentrations of $\sim 4 \times 10^{5} \mathrm{~cm}^{-3}$. During the formation of the $\mathrm{CN}$ layer, however, there are more preexisting $\mathrm{CN}$ present that reduce the nucleation efficiency and therefore ion-induced nucleation only starts to occur at sulfuric acid concentrations of $\sim 6 \times 10^{5} \mathrm{~cm}^{-3}$. As the derived sulfuric acid concentrations are below that limit in September, the CN layer formation is not influenced significantly by ion-induced nucleation. In October, the $\mathrm{CN}$ layer is located mostly in the area where ions do change the nucleation rates, but as there is no nucleation of new particles during that time, the derived sulfuric acid hardly changes by ion-induced nucleation.

\subsection{Sensitivity studies}

To study the uncertainty introduced by the assumed particle size threshold of the $\mathrm{CN}$ measurements, we derive the sulfu- 

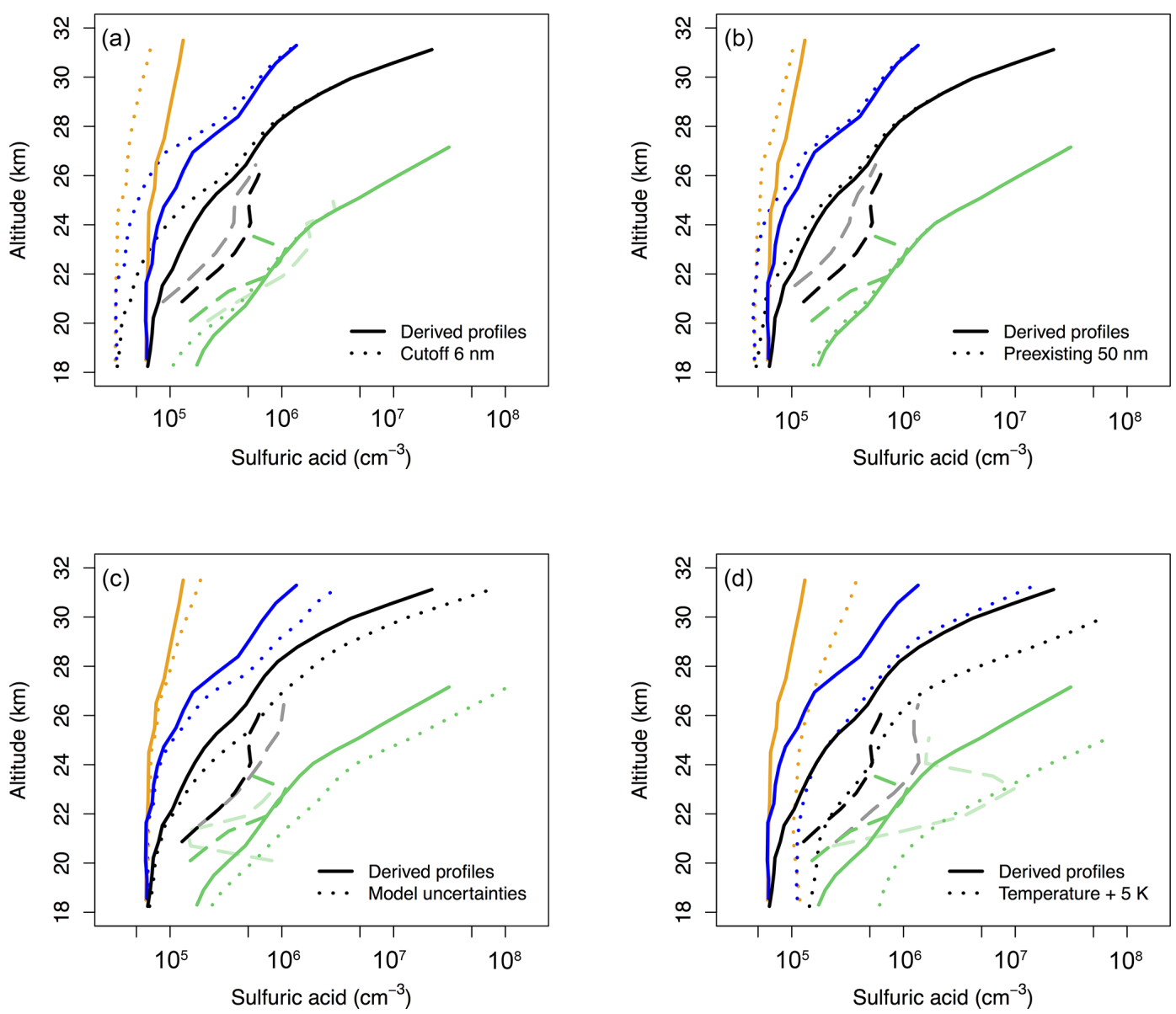

Figure 6. Sensitivity studies varying (a) CN counter cutoff size, (b) preexisting particle size, (c) model thermochemical and dynamic parameters, and (d) temperature to estimate the uncertainties of the derived sulfuric acid profiles. As in Fig. 5, the solid and dark dashed lines show the nucleation threshold and $\mathrm{CN}$ layer formation profiles as presented in Fig. 4. The dotted and light dashed lines show the changed profiles according to the sensitivity tests.

ric acid profiles assuming a lower $\mathrm{CN}$ counter threshold of $6 \mathrm{~nm}$ diameter, which is the $\mathrm{CN}$ counter's lower end according to Campbell and Deshler (2014). The lower threshold leads to lower sulfuric acid concentrations as the nucleated $\mathrm{CN}$ do not have to grow as large by sulfuric acid condensation to be counted (Fig. 6a). The impact of $\mathrm{CN}$ threshold size decreases with increasing sulfuric acid as, at higher concentrations, the clusters grow quickly once they are nucleated. In October, however, there is more sulfuric acid needed in the $\mathrm{CN}$ layer as fewer small clusters exist that can grow across the cutoff size and therefore some nucleation of new $\mathrm{CN}$ is needed.

Lowering the size of the initial preexisting particles from 100 to $50 \mathrm{~nm}$ diameter reduces their coagulation efficiency and they present a smaller loss during nucleation. Therefore, the modeled sulfuric acid concentrations are lower (Fig. 6b). For the same reason there is no sulfuric acid needed in October in the $\mathrm{CN}$ layer. If we assume the initial preexisting particles to be a distribution of different sizes (e.g., $40 \%$ of
$50 \mathrm{~nm}, 50 \%$ of $100 \mathrm{~nm}$, and $10 \%$ of $300 \mathrm{~nm}$ particles), the coagulation efficiency increases and leads to fewer simulated $\mathrm{CN}$ and higher derived sulfuric acid profiles (Fig. S1 in the Supplement).

We study model uncertainties according to Lovejoy et al. (2004) by adding $0.5 \mathrm{kcal}$ to all changes in Gibbs free energy of negatively charged clusters. This only increases the profiles in regions where ion-induced nucleation dominates (see Sect. 3.2 and Fig. 5). A reduction of all coagulation and condensation rates by $20 \%$ increases all profiles a little but leads to a poorer $\mathrm{CN}$ simulation in comparison with the observations. The updated neutral sulfuric acid dimer thermodynamic stabilities presented by Kürten et al. (2015), which have a higher relative humidity dependence of the equilibrium constant, lead to higher dimer evaporation rates. Therefore, they increase our profiles at low relative humidities (high temperatures), but only if neutral binary nucleation dominates. A combination of these influences is shown in Fig. 6c. The increase in the September CN layer profile at 


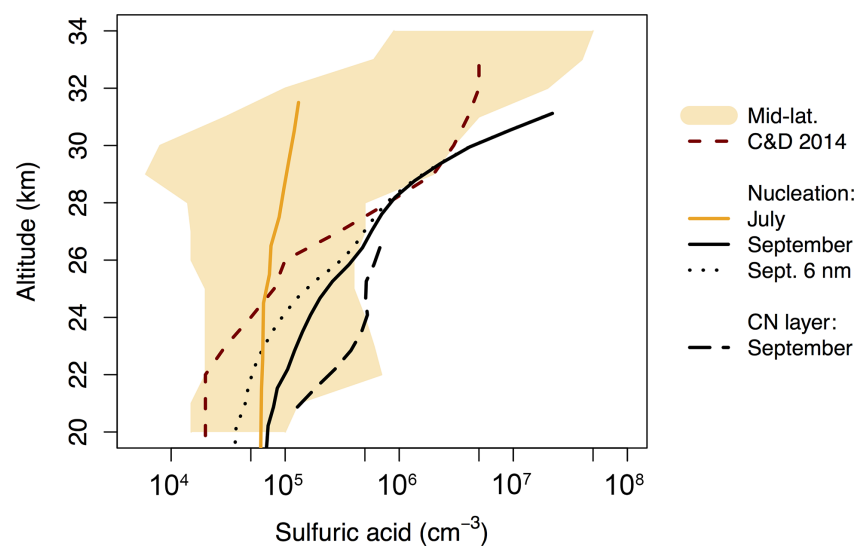

Figure 7. Comparison of our derived Antarctic sulfuric acid profiles (nucleation threshold: solid; $\mathrm{CN}$ layer: long dashed) with the derived profile from Campbell and Deshler (2014) (dark red, short dashed) and midlatitude measurements and modeling of Arnold et al. (1981), Reiner and Arnold (1997), Schlager and Arnold (1987), Viggiano and Arnold (1981), and Mills et al. (2005) (shaded area). The September nucleation threshold profile for nucleation and growth to a lower cutoff of $6 \mathrm{~nm}$ from Fig. 6a is also included (black dotted).

$24-26 \mathrm{~km}$ is mainly due to the updated dimer thermodynamic stabilities. The October $\mathrm{CN}$ layer profile mostly decreases as coagulation is less efficient, which requires less growth of additional small particles. At the lowest altitude no nucleation is needed in September, but therefore nucleation of additional $\mathrm{CN}$ is necessary in October.

As the derived sulfuric acid profiles are mainly determined by temperature we also test the effect of a $5 \mathrm{~K}$ temperature increase (Fig. 6d). We removed the responses at the highest September and October values as there the temperature was too high, so that evaporating particles complicate the situation. A $5 \mathrm{~K}$ temperature increase significantly increases the sulfuric acid profiles by a factor of 2 in the coldest regions and up to a factor of 15 in the warmest regions. Fortunately, the temperature measurement uncertainty is only $0.5 \mathrm{~K}$ (Campbell and Deshler, 2014). However, this temperature sensitivity shows that our sulfuric acid profiles in July and August at low altitudes are up to a factor of 2 too high as there we had to increase the temperature to SAWNUC's lower temperature limit of $190 \mathrm{~K}$ (maximum increase of $5 \mathrm{~K}$; see Fig. 1a).

Our trajectories might descend too fast from July to August as the CN profile of Campbell and Deshler (2014) is representative of June and July. Also, Campbell and Deshler (2014) note that most measurements were performed between late August and early October, while our October simulations reproduce the measured $\mathrm{CN}$ as a monthly mean. If we run our simulations from mid-June until mid-October, the simulated $\mathrm{CN}$ in August are lower as the preexisting $\mathrm{CN}$ have more time to coagulate and in October less sulfuric acid is necessary to reproduce the $\mathrm{CN}$ layer (Fig. S2). Note that, in combination with a preexisting particle size distribution, this might necessitate some nucleation already in August.

Additional sensitivity studies (Fig. S3) imply that the exact number of ions or water molecules (e.g., 5 ppm everywhere) has only a small influence on the derived profiles because the ion concentrations are high enough that they are not a limiting factor, and the few parts per million stratospheric water vapor uncertainty is too small to influence the profiles significantly. Also, a formation of $35 \%$ more $\mathrm{CN}$ in the layer $(\mathrm{CN}$ measurement uncertainty) needs only little additional sulfuric acid (not shown).

\subsection{Comparison with midlatitude sulfuric acid and the derived profile of Campbell and Deshler (2014)}

In Fig. 7 we compare our derived September CN layer sulfuric acid profile with the profile derived by Campbell and Deshler (2014). Campbell and Deshler (2014) derived sulfuric acid concentrations for 15 to $33 \mathrm{~km}$ (dark red, dashed). Our derived sulfuric acid (black, dashed) is only shown between 21 and $26 \mathrm{~km}$ as we need no nucleation above and below the $\mathrm{CN}$ layer to reproduce the observations. Our concentrations are about 1 order of magnitude higher. This is because our $\mathrm{CN}$ have to form in a background of preexisting particles and they have to grow to observable size. As our sensitivity tests show, both of these effects require more sulfuric acid. In the nucleation threshold profile with a cutoff of $6 \mathrm{~nm}$ and a background of $10 \mathrm{CN} \mathrm{cm}^{-3}$ (black, dotted) these two effects are less pronounced, and therefore this profile compares better with the derived profile of Campbell and Deshler (2014).

We cannot compare our derived sulfuric acid profiles with Antarctic in situ or remote sensing measurements as such data do not exist to our knowledge. However, northern midlatitude balloon-borne measurements mainly from September and October have been published (Arnold et al., 1981; Reiner and Arnold, 1997; Schlager and Arnold, 1987; Viggiano and Arnold, 1981) and summarized by Mills et al. (2005). Note that due to the different tropopause heights $\left(43^{\circ} \mathrm{N}\right.$ vs. $\left.78^{\circ} \mathrm{S}\right)$ our derived profiles might need to be shifted upwards for comparison. In July and August our nucleation threshold profiles lie within the midlatitude values. However, as nucleation does not occur, these profiles represent upper limits and Antarctic winter sulfuric acid concentrations are lower than average midlatitude concentrations. In September our derived sulfuric acid concentrations in the $\mathrm{CN}$ layer are higher than average midlatitude concentrations. This comparison supports the formation explanation of the $\mathrm{CN}$ layer with low sulfuric acid during Antarctic winter followed by an area of high sulfuric acid after sunrise.

We did not derive sulfuric acid profiles above Wyoming according to Fig. 1a and b of Campbell and Deshler (2014), as these $\mathrm{CN}$ are assumed to have nucleated in the polar region. However, as temperature mainly controls the nucleation rate, the nucleation threshold sulfuric acid profiles at tem- 
peratures representative of the stratosphere above Wyoming are used for comparison. In autumn, temperatures above Wyoming lie between $-60^{\circ} \mathrm{C}$ at $17 \mathrm{~km}$ and $-40^{\circ} \mathrm{C}$ at $34 \mathrm{~km}$ (Campbell and Deshler, 2014, Fig. 1b). The same temperature range is found over Antarctica in September between 27 and $33 \mathrm{~km}$ (Campbell and Deshler, 2014, Fig. 1d). If we compare our September nucleation threshold profile (black, solid) between 27 and $33 \mathrm{~km}$ with the midlatitude values, we see that at all midlatitude altitudes the concentrations are just below our nucleation threshold values. This suggests that nucleation usually does not occur in the midlatitude stratosphere in this altitude range. Sometimes at the highest sulfuric acid levels, however, ion-induced nucleation may become efficient.

\section{Summary and conclusions}

Analysis of over 20 years (1986-2010) of balloon-borne stratospheric CN measurements above McMurdo Station, Antarctica, between July and October reveals the formation of a layer of mainly volatile $\mathrm{CN}$ at $21-27 \mathrm{~km}$ altitude in a background of preexisting particles (Campbell and Deshler, 2014). Here, we use the nucleation box model SAWNUC to simulate these $\mathrm{CN}$ in subsiding air parcels and study the nucleation processes.

The observed CN of Campbell and Deshler (2014) are reproduced by simulating subsiding air parcels with volume compression, coagulation, nucleation, and growth processes. Antarctic $\mathrm{CN}$ concentrations outside the $\mathrm{CN}$ layer can be explained by coagulation if air volume compression due to air parcel subsidence is considered. Neutral sulfuric acid-water nucleation forms the CN layer in September, while in October growth of small particles maintains the layer. Ion-induced nucleation does not occur at significant levels as sulfuric acid concentrations are too low and charged clusters recombine too fast. Our results complement Campbell and Deshler (2014), who showed that the $\mathrm{CN}$ decrease above Laramie, Wyoming, can be explained by coagulation and that almost all $\mathrm{CN}$ inside the $\mathrm{CN}$ layer are volatile and therefore can be explained by binary nucleation.

Sulfuric acid concentrations in September during the CN layer formation range from $1 \times 10^{5} \mathrm{~cm}^{-3}$ at $21 \mathrm{~km}$ to $6 \times$ $10^{5} \mathrm{~cm}^{-3}$ at $26 \mathrm{~km}$, which is about 1 order of magnitude higher than the concentrations derived by Campbell and Deshler (2014). Our sensitivity tests show that more sulfuric acid is needed in our simulations because nucleated clusters have to grow to the $\mathrm{CN}$ counter's threshold size for detection and can coagulate with preexisting particles. Therefore, we can confirm the suggestion of Campbell and Deshler (2014) that their profiles might be an underestimation due to these effects.

Finally, we derived gaseous sulfuric acid profiles that show which concentration would be necessary for nucleation and growth to $\mathrm{CN}$ size to occur, which should represent upper limits of the actual sulfuric acid outside of the $\mathrm{CN}$ layer where neither the observations nor our simulations indicate nucleation to occur. The upper limits start at $18 \mathrm{~km}$ at concentrations below $10^{5} \mathrm{~cm}^{-3}$ and increase to about $10^{5} \mathrm{~cm}^{-3}$ in July, $10^{6} \mathrm{~cm}^{-3}$ in August, and $10^{7} \mathrm{~cm}^{-3}$ in September at $32 \mathrm{~km}$, while in October they start at $2 \times 10^{5} \mathrm{~cm}^{-3}$ at $18 \mathrm{~km}$ and increase above $10^{7} \mathrm{~cm}^{-3}$ at $27 \mathrm{~km}$. According to these upper limits, sulfuric acid concentrations seem to be below midlatitude average during Antarctic winter but above midlatitude average during the $\mathrm{CN}$ layer formation, while midlatitude sulfuric acid concentrations in general seem to be too low for nucleation to occur. This is also in agreement with Campbell and Deshler (2014) and other seminal references contained within, who suggest that the midlatitude $\mathrm{CN}$ layer originally formed in the polar region.

If stratospheric sulfuric acid increases above our upper limits, e.g., because of volcanic eruptions or geoengineering, nucleation could occur. In the midlatitudes and in some relatively warm areas above Antarctica, this nucleation would be dominated by ion-induced nucleation and therefore would require less sulfuric acid than predicted by neutral binary nucleation theory. Note, however, that our upper limits would increase if there were more preexisting particles present.

In conclusion, our study supports the explanation of the CN layer as presented by Campbell and Deshler (2014). We can reproduce the $\mathrm{CN}$ that decrease over time by coagulation in a low sulfuric acid environment during Antarctic winter. In September between 21 and $26 \mathrm{~km}$ we can reproduce the observed CN layer only if we assume a higher sulfuric acid concentration that produces volatile $\mathrm{CN}$ mainly by neutral binary nucleation.

Data availability. The data shown in the figures are available from the authors upon request.

\section{The Supplement related to this article is available online at https://doi.org/10.5194/acp-17-7581-2017-supplement.}

Competing interests. The authors declare that they have no conflict of interest.

Acknowledgements. We thank Edward R. Lovejoy, Karl D. Froyd, Jan Kazil, and Sebastian Ehrhart for providing the SAWNUC code and Andreas Engel for useful discussion. We thank the two anonymous reviewers for numerous helpful comments.

Edited by: Holger Tost

Reviewed by: two anonymous referees 


\section{References}

Arnold, F., Fabian, R., and Joos, W.: Measurements of the height variation of sulfuric acid vapor concentrations in the stratosphere. Geophys. Res. Lett., 8, 293-296, 1981.

Arnold, F., Curtius, J., Spreng, S., and Deshler, T.: Stratospheric aerosol sulfuric acid: First direct in situ measurements using a novel balloon-based mass spectrometer apparatus, J. Atmos. Chem., 30, 3-10, 1998.

Borrmann, S., Kunkel, D., Weigel, R., Minikin, A., Deshler, T., Wilson, J. C., Curtius, J., Volk, C. M., Homan, C. D., Ulanovsky, A., Ravegnani, F., Viciani, S., Shur, G. N., Belyaev, G. V., Law, K. S., and Cairo, F.: Aerosols in the tropical and subtropical UT/LS: in-situ measurements of submicron particle abundance and volatility, Atmos. Chem. Phys., 10, 5573-5592, https://doi.org/10.5194/acp-10-5573-2010, 2010.

Brock, C. A., Hamill, P., Wilson, J. C., Jonsson, H. H., and Chan, K. R.: Particle formation in the upper tropical troposphere: A source of nuclei for the stratospheric aerosol, Science, 270, p. 1650, 1995.

Campbell, P. and Deshler, T.: Condensation nuclei measurements in the midlatitude (1982-2012) and Antarctic (1986-2010) stratosphere between 20 and $35 \mathrm{~km}$, J. Geophys. Res.-Atmos., 119, 137-152, 2014.

Campbell, P., Mills, M., and Deshler, T.: The global extent of the mid stratospheric CN layer: A three-dimensional modeling study, J. Geophys. Res.-Atmos., 119, 1010-1030, 2014.

Chan, T. W. and Mozurkewich, M.:. Measurement of the coagulation rate constant for sulfuric acid particles as a function of particle size using tandem differential mobility analysis, J. Aerosol Sci., 32, 321-339, 2001.

Curtius, J., Weigel, R., Vössing, H.-J., Wernli, H., Werner, A., Volk, C.-M., Konopka, P., Krebsbach, M., Schiller, C., Roiger, A., Schlager, H., Dreiling, V., and Borrmann, S.: Observations of meteoric material and implications for aerosol nucleation in the winter Arctic lower stratosphere derived from in situ particle measurements, Atmos. Chem. Phys., 5, 3053-3069, https://doi.org/10.5194/acp-5-3053-2005, 2005.

Deshler, T.: A review of global stratospheric aerosol: Measurements, importance, life cycle, and local stratospheric aerosol, Atmos. Res., 90, 223-232, 2008.

Ehrhart, S. and Curtius, J.: Influence of aerosol lifetime on the interpretation of nucleation experiments with respect to the first nucleation theorem, Atmos. Chem. Phys., 13, 11465-11471, https://doi.org/10.5194/acp-13-11465-2013, 2013.

Ehrhart, S., Ickes, L., Almeida, J., Amorim, A., Barmet, P., Bianchi, F., Dommen, J., Dunne, E. M., Duplissy, J., Franchin, A., Kangasluoma, J., Kirkby, J., Kürten, A., Kupc, A., Lehtipalo, K., Nieminen, T., Riccobono, F., Rondo, L., Schobesberger, S., Steiner, G., Tomé, A., Wimmer, D., Baltensperger, U., Wagner, P. E., and Curtius, J.: Comparison of the SAWNUC model with CLOUD measurements of sulphuric acid-water nucleation, J. Geophys. Res.-Atmos., 121, https://doi.org/10.1002/2015JD023723, 2016.

English, J. M., Toon, O. B., Mills, M. J., and Yu, F.: Microphysical simulations of new particle formation in the upper troposphere and lower stratosphere, Atmos. Chem. Phys., 11, 9303-9322, https://doi.org/10.5194/acp-11-9303-2011, 2011.

Froyd, K. D. and Lovejoy, E. R.: Experimental thermodynamics of cluster ions composed of $\mathrm{H}_{2} \mathrm{SO}_{4}$ and $\mathrm{H}_{2} \mathrm{O}$. 2. Measurements and ab initio structures of negative ions, J. Phys. Chem. A, 107, 9812-9824, 2003a.

Froyd, K. D. and Lovejoy, E. R.: Experimental thermodynamics of cluster ions composed of $\mathrm{H}_{2} \mathrm{SO}_{4}$ and $\mathrm{H}_{2} \mathrm{O}$. 1. Positive ions, J. Phys. Chem. A, 107, 9800-9811, 2003b.

Hamill, P., Toon, O. B., and Turco, R. P.: Aerosol nucleation in the winter Arctic and Antarctic stratospheres, Geophys. Res. Lett., 17, 417-420, 1990.

Hanson, D. R. and Lovejoy, E. R.: Measurement of the thermodynamics of the hydrated dimer and trimer of sulfuric acid, J. Phys. Chem. A, 110, 9525-9528, 2006.

Hardiman, S. C., Butchart, N., and Calvo, N.: The morphology of the Brewer-Dobson circulation and its response to climate change in CMIP5 simulations, Q. J. Roy. Meteor. Soc., 140, 1958-1965, 2014.

Hofmann, D. J.: Measurement of the condensation nuclei profile to $31 \mathrm{~km}$ in the Arctic in January 1989 and comparisions with Antarctic measurements, Geophys. Res. Lett., 17, 357-360, 1990.

Hofmann, D. J. and Rosen, J. M.: Antarctic observations of stratospheric aerosol and high altitude condensation nuclei following the El Chichon eruption, Geophys. Res. Lett., 12, 13-16, 1985.

Hurrell, J. W., Holland, M. M., Gent, P. R., Ghan, S., Kay, J. E., Kushner, P. J., Lamarque, J.-F., Large, W. G., Lawrence, D., Lindsay, K., Lipscomb, W. H., Long, M. C., Mahowald, N., Marsh, D. R., Neale, R. B., Rasch, P., Vavrus, S., Vertenstein, M., Bader, D., Collins, W. D., Hack, J. J., Kiehl, J., and Marshall, S.: The community earth system model: A framework for collaborative research, B. Am. Meteorol. Soc., 94, 1339-1360, 2013.

Junge, C. E., Chagnon, C. W., and Manson, J. E.: Stratospheric aerosols, J. Meteorol., 18, 81-108, 1961.

Kazil, J. and Lovejoy, E. R.: A semi-analytical method for calculating rates of new sulfate aerosol formation from the gas phase, Atmos. Chem. Phys., 7, 3447-3459, https://doi.org/10.5194/acp7-3447-2007, 2007.

Kirkby, J., Kirkby, J., Curtius, J., Almeida, J., Dunne, E., Duplissy, J., Ehrhart, S., Franchin, A., Gagné, S., Ickes, L., Kürten, A., Kupc, A., Metzger, A., Riccobono, F., Rondo, L., Schobesberger, S., Tsagkogeorgas, G., Wimmer, D., Amorim, A., Bianchi, F., Breitenlechner, M., David, A., Dommen, J., Downard, A., Ehn, M., Flagan, R. C., Haider, S., Hansel, A., Hauser, D., Jud, W., Junninen, H., Kreissl, F., Kvashin, A., Laaksonen, A., Lehtipalo, K., Lima, J., Lovejoy, E. R., Makhmutov, V., Mathot, S., Mikkilä, J., Minginette, P., Mogo S., Nieminen, T., Onnela, A., Pereira, P., Petäjä, T., Schnitzhofer, R., J. H. Seinfeld, Sipilä, M., Stozhkov, Y., Stratmann, F., Tomé, A., Vanhanen, J., Viisanen, Y., Vrtala, A., Wagner, P. E., Walther, H., Weingartner, E., Wex, H., Winkler, P. M., Carslaw, K. S., Worsnop, D. R., Baltensperger, U., and Kulmala, M.: Role of sulphuric acid, ammonia and galactic cosmic rays in atmospheric aerosol nucleation, Nature, 476, 429-433, 2011.

Kürten, A., Münch, S., Rondo, L., Bianchi, F., Duplissy, J., Jokinen, T., Junninen, H., Sarnela, N., Schobesberger, S., Simon, M., Sipilä, M., Almeida, J., Amorim, A., Dommen, J., Donahue, N. M., Dunne, E. M., Flagan, R. C., Franchin, A., Kirkby, J., Kupc, A., Makhmutov, V., Petää, T., Praplan, A. P., Riccobono, F., Steiner, G., Tomé, A., Tsagkogeorgas, G., Wagner, P. E., Wimmer, D., Baltensperger, U., Kulmala, M., Worsnop, 
D. R., and Curtius, J.: Thermodynamics of the formation of sulfuric acid dimers in the binary $\left(\mathrm{H}_{2} \mathrm{SO}_{4}-\mathrm{H}_{2} \mathrm{O}\right)$ and ternary $\left(\mathrm{H}_{2} \mathrm{SO}_{4}-\mathrm{H}_{2} \mathrm{O}-\mathrm{NH}_{3}\right)$ system, Atmos. Chem. Phys., 15, 1070110721, https://doi.org/10.5194/acp-15-10701-2015, 2015.

Lovejoy, E. R. and Curtius, J.: Cluster ion thermal decomposition (II): Master equation modeling in the low-pressure limit and falloff regions. Bond energies for $\mathrm{HSO}_{4}-\left(\mathrm{H}_{2} \mathrm{SO}_{4}\right) \times\left(\mathrm{HNO}_{3}\right) \mathrm{y}, \mathrm{J}$. Phys. Chem. A, 105, 10874-10883, 2001.

Lovejoy, E. R., Curtius, J., and Froyd, K. D.: Atmospheric ioninduced nucleation of sulfuric acid and water, J. Geophys. Res.Atmos., 109, https://doi.org/10.1029/2003JD004460, 2004.

McMurry, P. H.: The history of condensation nucleus counters, Aerosol Sci. Tech., 33, 297-322, 2000.

Mills, M. J., Toon, O. B., and Solomon, S.: A 2D microphysical model of the polar stratospheric CN layer, Geophys. Res. Lett., 26, 1133-1136, 1999.

Mills, M. J., Toon, O. B., Vaida, V., Hintze, P. E., Kjaergaard, H. G., Schofield, D. P., and Robinson, T. W.: Photolysis of sulfuric acid vapor by visible light as a source of the polar stratospheric CN layer, J. Geophys. Res.-Atmos., 110, https://doi.org/10.1029/2004JD005519, 2005.

Murphy, D. M., Thomson, D. S., and Mahoney, M. J.: In situ measurements of organics, meteoritic material, mercury, and other elements in aerosols at 5 to 19 kilometers. Science, 282, 1664$1669,1998$.

Murphy, D. M., Froyd, K. D., Schwarz, J. P., and Wilson, J. C.: Observations of the chemical composition of stratospheric aerosol particles, Q. J. Roy. Meteor. Soc., 140, 1269-1278, 2014.

Reiner, T. and Arnold, F.: Stratospheric $\mathrm{SO}_{3}$ : Upper limits inferred from ion composition measurements-Implications for $\mathrm{H}_{2} \mathrm{SO}_{4}$ and aerosol formation, Geophys. Res. Lett., 24, 1751-1754, 1997.

Rosen, J. M. and Hofmann, D. J.: Unusual behavior in the condensation nuclei concentration at $30 \mathrm{~km}$, J. Geophys. Res.-Oceans, 88, 3725-3731, 1983.

Schlager, H. and Arnold, F.: Balloon-borne composition measurements of stratospheric negative ions and inferred sulfuric acid vapor abundances during the MAP/GLOBUS 1983 campaign, Planet. Space Sci., 35, 693-701, 1987.

Thomason, L. and Peter, T.: SPARC assessment of stratospheric aerosol properties, WCRP-124, WMO/TD-No. 1295 (No. 4), SPARC Report, 2006.
Usoskin, I. G., Bazilevskaya, G. A., and Kovaltsov, G. A.: Solar modulation parameter for cosmic rays since 1936 reconstructed from ground-based neutron monitors and ionization chambers, J. Geophys. Res.-Space, 116, https://doi.org/10.1029/2010JA016105, 2011.

Vaida, V., Kjaergaard, H. G., Hintze, P. E., and Donaldson, D. J.: Photolysis of sulfuric acid vapor by visible solar radiation, Science, 299, 1566-1568, 2003.

Viggiano, A. A. and Arnold, F.: Extended sulfuric acid vapor concentration measurements in the stratosphere, Geophys. Res. Lett., 8, 583-586, 1981.

Vömel, H., Oltmans, S. J., Hofmann, D. J., Deshler, T., and Rosen, J. M.: The evolution of the dehydration in the Antarctic stratospheric vortex, J. Geophys. Res.-Atmos., 100, 13919-13926, 1995.

Waters, J. W., Froidevaux, L., Harwood, R. S., Jarnot, R. F., Pickett, H. M., Read, W. G., Siegel, P. H., Cofield, R. E., Filipiak, M. J., Flower, D. A., Holden, J. R., Lau, G. K., Livesey, N. J., Manney, G. L., Pumphrey, H. C., Santee, M. L., Wu, D. L., Cuddy, D. T., Lay, R. R., Loo, M. S., Perun, V. S., Schwartz, M. J., Stek, P. C., Thurstans, R. P., Boyles, M. A., Chandra, K. M., Chavez, M. C., Chen, G.-S., Chudasama, B. V., Dodge, R., Fuller, R. A., Girard, M. A., Jiang, J. H., Jiang, Y., Knosp, B. W., LaBelle, R. C., Lam, J. C., Lee, K. A., Miller, D., Oswald, J. E., Patel, N. C., Pukala, D. M., Quintero, O., Scaff, D. M., Van Snyder, W., Tope, M C., Wagner, P. A., and Walch, M. J.: The earth observing system microwave limb sounder (EOS MLS) on the Aura satellite, IEEE T. Geosci. Remote Sens., 44, 1075-1092, 2006.

Weigel, R., Borrmann, S., Kazil, J., Minikin, A., Stohl, A., Wilson, J. C., Reeves, J. M., Kunkel, D., de Reus, M., Frey, W., Lovejoy, E. R., Volk, C. M., Viciani, S., D’Amato, F., Schiller, C., Peter, T., Schlager, H., Cairo, F., Law, K. S., Shur, G. N., Belyaev, G. V., and Curtius, J.: In situ observations of new particle formation in the tropical upper troposphere: the role of clouds and the nucleation mechanism, Atmos. Chem. Phys., 11, 9983-10010, https://doi.org/10.5194/acp-11-9983-2011, 2011.

Zhao, J., Toon, O. B., and Turco, R. P.: Origin of condensation nuclei in the springtime polar stratosphere, J. Geophys. Res.Atmos., 100, 5215-5227, 1995. 